\title{
MILP-based heuristics for the design of rural community electrification projects
}

\author{
Joan Triadó-Aymerich ${ }^{\mathrm{a}}$, Laia Ferrer-Martí ${ }^{\mathrm{b}}$, Alberto García-Villoria ${ }^{\mathrm{b} *}$, Rafael Pastor ${ }^{\mathrm{b}}$ \\ a Escola Superior Politècnica de Mataró, Pompeu Fabra University,, C/ Ernest Lluch 32, Mataró, Spain \\ ${ }^{\mathrm{b}}$ Institute of Industrial and Control Engineering, Universitat Politècnica de Catalunya- Barcelona Tech (UPC), Av. \\ Diagonal 647, Barcelona, Spain
}

\begin{abstract}
Wind-photovoltaic systems are a suitable option to provide electricity to isolated communities autonomously. To design these systems, there are recent mathematical models that solve the location and type of each of the electrification components and the design of the possible distribution microgrids. When the amount of demand points to electrify increases, solving the mathematical model requires a computational time that become infeasible in practice. To speed up the solving process, three heuristic methods based on mixed integer linear programming (MILP) are presented in this paper: Relax and Fix heuristics, heuristics based on Corridor Method and Increasing Radius heuristics. In all algorithms first a relaxed MILP is solved to obtain a base solution and then it is used as a starting point to find a feasible solution by searching in a reduced search space. For each type of heuristic several options to relax and to reduce the solution space are developed and tested. Extensive computational experiments based on real projects are carried out and results show that the best heuristic vary according to the size of instances.
\end{abstract}

Keywords: MILP, heuristics, rural electrification, wind-photovoltaic systems

\section{Nomenclature}

- Demand points: location of consumption points (users), such as houses or public buildings to electrify.

- Microgrid: Set of demand points connected to each other and fed by the same generation system placed at one of the demand points.

- Community: the group of users.

- Connection: the existence of cable (regardless its type) between two demand points.

\section{Introduction}

Over 1.3 billion people worldwide lack access to electricity, especially in rural areas of developing countries [1]. In some regions access to conventional electricity grid is very difficult due to the complexity of the terrain and the dispersal of populations. In these cases, a suitable alternative to provide contact to electricity are autonomous systems based on the use of renewable energy sources [2, 3] since they promote the sustainability of the projects due to the handling of local sources thereby avoid external dependencies, and tend to be cheaper than the extension of the conventional electricity grid.

Photovoltaic systems (PV) have been widely used in recent decades to electrify rural communities. On the other hand, wind systems are increasingly being used [4, 5, e.g.]. Compared with PV, in windy regions the use of wind systems may be more profitable, especially if demand increases and powerful turbines are used. On the other hand, hybrid systems are more efficient, providing more supply reliability and consequently, requiring less energy storage if compared with single energy resource systems [6, 7].

In autonomous electrification networks, individual systems at each demand point are typically used [8]. However, microgrid distribution arrangements, in which energy is produced in a specific point and distributed by the electric grid to other demand points, have advantages [9, 10]. Its implementation could imply significant savings in the investment cost compared with individual systems, taking advantage of the best resource areas and economies of scale. The combination of microgrids and individual systems (points individually fed) may be the best solution in dispersed communities and it was incorporated in [11].

Due to the complexity of designing hybrid systems, in the last years different methods to assist designers have appeared. Most of them are focused exclusively on the definition of the best combination of energy resources [12-15] but not on the distribution grid design. Others consider both problems although all the points must be connected to one

\footnotetext{
* Corresponding author: Alberto García-Villoria, Universitat Politècnica de Catalunya- Barcelona Tech (UPC), Av. Diagonal 647, 08028 Barcelona, Spain; E-mail: alberto.garcia-villoria@upc.edu
} 
only grid [16-18]. There are few references that deal with autonomous electrification systems designs using hybrid systems and microgrids or individual systems at the same time [11, 19, 20].

The use of optimization methods is increasingly a powerful tool for solving real-life problems as the design of electrification systems using renewable energies [21, 22]. Especially, linear programming has been widely used in recent years [23-25] on the conception of energy systems. A MILP model to scheme wind electrification projects that combine microgrids and individual systems has been presented in [20]. Afterwards, a new model was developed in [11] incorporating solar generation. The last aforementioned model has been used in projects at Peru [5] and Cape Verde [26] in order to optimize the design of all the components of autonomous electrification systems: solar panels and wind turbines, electric equipment and cables. The model considers voltage drops and the variability of wind resource according to the placing of generation in demand points.

However, the application of mathematical models to solve this kind of hard combinatorial optimization problems (COPs) is not practical when the size of instances increases, since they spend high computational times. For that reason, heuristics are commonly used to solve COPs [27] in order to obtain solutions reasonably close to the optimal or near optimal solutions in a practical time. For instance VIPOR is a simulated annealing method which designs autonomous electrification projects considering hybrid generation, microgrids and spatial variation of resources [19, 28]. ViPOR determines the location of generation and the distribution grid but with some technical limitations; in particular, it assumes uniform resource in all the points for individual generation and does not calculate voltage drops. A recent study has been presented developing a set of indicators to support the design of autonomous electrification projects [29]. These indicators can be used to select the potential generation points. Furthermore, it evaluates heuristically the suitability of the points to be (or not) connected to a microgrid. A heuristic is also presented in [29] to obtain quickly single-microgrid solutions; i.e., solutions constrained to no more than one microgrid.

To the best of our knowledge, heuristic methods based on MILP have not been applied to autonomous electrification projects although they have been applied successfully in other kind of grid systems problems [30, 31]. In this paper we present heuristic methods based on a MILP model to solve the problem of autonomous electrification systems formed by microgrids powered by solar and wind generators located at one of the points in each microgrid, and allowing the possibility of containing individual systems. The following three type of MILP-based heuristics are presented: Relax and Fix heuristics, Heuristics based on Corridor Method and adhoc developed heuristics that we call Increasing Radius. Instances with high number of demand points are tackled in this work and results show the best heuristic to use depending on the size of the instance.

The paper is organized as follows. Section 2 presents the problem and the components of the autonomous electrification project. Section 3 describes the MILP model in which the proposed heuristics are based. In section 4 the heuristics are presented. In section 5, computational experiments are carried out to evaluate and compare the performance of the algorithms. Finally, Section 6 concludes this paper.

\section{Rural autonomous electrification project}

The rural autonomous electrification project presented in this paper considers wind and solar generation placed in demand points. The design of these systems must take account of the availability of these energies in the region (wind resource maps and irradiation data). The electricity distribution combines individual systems and microgrids. The problem is to define which demand points will be included in each microgrid, or whether they will be individually fed; where generators and other components will be placed, and which and how many components are to be used, so as to minimize the initial investment satisfying the demand together with other technical constraints. 


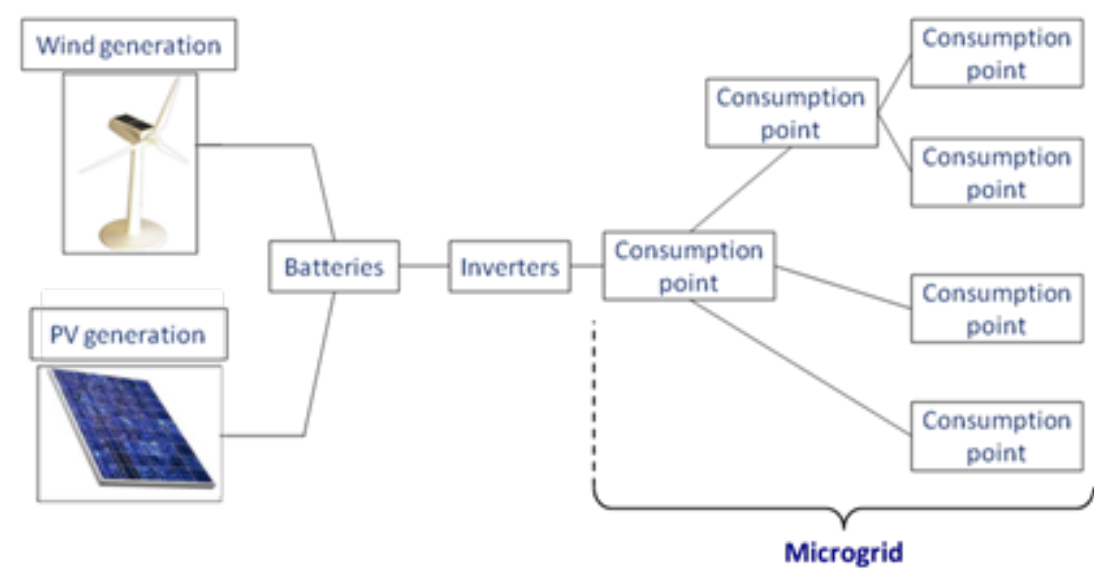

Figure 1: Scheme of rural autonomous electrification systems [11]

The components of the autonomous electrification project (Figure 1) are, first, the wind or photovoltaic (PV) generation equipment, which include the generators and the regulators. Next, are the batteries to store energy and the inverters to create alternating current (AC). Finally, there are the cables that distribute the electricity with radial microgrids [32] and meters at the demand points (e.g., houses) for measuring for measuring energy consumed. Each component of rural electrification project can be of different types in order to adequate the design to the needs of each zone.

The energy generated by a wind generator of a particular type depends on the wind resource at a specific demand point. On the other hand, the energy produced by a PV generator (solar panel) of a particular type does not depend on its location because the solar resource is considered uniform in all parts of a community [33]. The number of solar and wind generators are limited at a specific point for space sake. Regulators that transform AC into direct current (DC) power and control charge and discharge batteries are required between generators and batteries. Regulators for wind generators are part of the generator system, whereas regulators for solar generation are independent devices. The generated energy is accumulated in battery banks and subsequently is distributed to the electric system. The capacity of these batteries is calculated depending on the demand and the autonomy required. To allow the consumption of electricity in AC, typical scenario of electric distribution grid, inverters with the necessary power must be installed. The cables connect the different points of every microgrid to supply the energy from the generating point to the demand points. Energy consumption meters and power ones are placed only into the demand points that are part of a microgrid in order to measure and allow fairer consumptions among different consumers.

\section{Mathematical programming model}

Our paper develops a MILP-based heuristics from the model presented in [11]. That model is here extended and adapted to develop the heuristic algorithms proposed here. In particular, a limit on the maximum allowed cable length between two points is eliminated to extend the space of solutions. Moreover, specific variables that represent the existence of these connections $\left(x c_{p q}\right)$ are added to be the base of the fixations in the heuristics. From now on, this new model will be named as AREMWS (autonomous rural electrification model with wind and solar generation).

The description of the model is next presented: firstly the description of data; secondly, the variables; and finally the objective function and the constraints.

\section{- Model Data}

Data are next introduced and classified according to informations to the demand, the storage, the grid definition, the wind generation, the solar generation and other equipment:

\section{- Data referred to the demand:}

D Set of demand points (houses, healthcare centers, schools, shops, etc.), which are also generation points candidates.

$L_{p q} \quad$ Distance [m] between point $p$ and point $q ; p \in D, q \in D \backslash\{p\}$.

$P D_{p}, E D_{p} \quad$ Power [W] and energy [Wh/day] demand of point $p$, respectively; $p \in D$.

$V B \quad$ Autonomy time [days] that the batteries must have.

\section{- Data referred to the storage:}

B Types of batteries. 
$D B \quad$ Discharge factor of the batteries [\%].

$C B_{b}, E B_{b} \quad$ Cost [\$] and equivalent capacity [Wh] of a battery of type $b$, respectively; $b=1, \ldots, B$.

- Data referred to the grid definition:

C Types of cables.

$C C_{c} \quad$ Cost $[\$ / \mathrm{m}]$ of cable of type c, including the infrastructure cost; $c=1, \ldots, C$.

$R C_{c}, I C_{c} \quad$ Resistance $[\Omega / \mathrm{m}]$ and maximum current $[\mathrm{A}]$ admitted for the cable type $c$, respectively; $c=1, \ldots, C$.

$V_{n}, V_{\min }, V_{\max }$ Nominal, minimum and maximum voltage admitted [V], respectively.

$c d t_{\max } \quad$ Maximum voltage drop admitted [fraction of unity] $=\left(V_{\max }-V_{\min }\right) / V_{n}$.

$\eta_{c a b} \quad$ Voltage drop efficiency [fraction of unity] $=1-c d t_{\max }$.

\section{- $\quad$ Data referred to wind generation:}

A, NA Types of wind turbines and an upper bound of the number of the wind turbines that are installed at a specific point, respectively.

$E A_{p a} \quad$ Generated energy [Wh/day] by a wind turbine placed at point $p$ of type $a ; p \in D, a=1, \ldots, A$.

$C A_{a} \quad$ Cost [\$] of a wind turbine of type $a ; a=1, \ldots, A$.

\section{- Data referred to solar generation:}

$S$, NS Types of solar panels and an upper bound of the number of solar panels that are installed at a specific point, respectively.

$E S_{s} \quad$ Generated energy [Wh/day] by a solar panel of type $s ; \mathrm{s}=1, \ldots, S$.

$C S_{s}, P S_{s} \quad$ Cost [\$] and maximum power [W] of a solar panel of type $s$, respectively; $s=1, \ldots, S$.

$R \quad$ Types of solar regulators.

$C R_{r}, P R_{r} \quad$ Cost [\$] and maximum power [W] of a solar regulator of type $r$, respectively; $r=1, \ldots, R$.

\section{- Data referred to other components:}

I, NI Types of inverters and an upper bound of the number of inverters that can be installed at a specific point, respectively.

$C I_{i}, P I_{i} \quad$ Cost [\$] and maximum power [W] of an inverter of type $i$, respectively; $i=1, \ldots, I$.

CM Cost of a meter unit [\$].

$\eta_{\text {bat }}, \eta_{\text {inv }} \quad$ Efficiency [fraction of unity] of the batteries and the inverters, respectively.

\section{- Variables}

The variables of the model are next presented:

\section{- Binary variables:}

$x_{p} \quad$ indicates whether any generator is installed at point $p$. If so, the value is 1 and 0 otherwise, $p \in D$.

$x c_{p q c} \quad$ indicates whether there is connection from point $p$ to point $q$ with a cable of type $c$. If so, the value is 1 and 0 otherwise; $p \in D, q \in D \backslash\{p\}, c=1, \ldots, C$.

$x c c_{p q} \quad$ indicates whether there is connection from point $p$ to point $q$ (regardless of its type of cable). If so, the value is 1 and 0 otherwise; $p \in D, q \in D \mid\{p\}$.

$x m_{p} \quad$ indicates whether a meter is installed at point $p$ (and, thus, point $p$ is part of a microgrid). If so, the value is 1 and 0 otherwise; $p \in D$.

\section{- Integer variables:}

$x a_{p a} \quad$ indicates the number of wind turbines with its corresponding regulator that is installed at the point $p$ of type $a ; p \in D, a=1, \ldots, A$.

$x s_{p s} \quad$ indicates the number of solar panels installed at point $p$ of type $s ; p \in D, s=1, \ldots, S$.

$x b_{p b} \quad$ indicates the number of batteries installed at point $p$ of type $b ; p \in D, b=1, \ldots, B$.

$x i_{p i} \quad$ indicates the number of inverters installed at point $p$ of type $i ; p \in D, i=1, \ldots, I$.

$x r s_{p r} \quad$ indicates the number of solar regulators installed at point $p$ of type $r ; p \in D, r=1, \ldots, R$.

\section{- Continuous variables:}

$f e_{p q} \quad$ indicates the energy flux [Wh/day] from point $p$ to point $q ; p \in D, q \in D \backslash\{p\}$.

$f p_{p q} \quad$ indicates the power flux [W] from point $p$ to point $q ; p \in D, q \in D \mid\{p\}$.

$v_{p} \quad$ indicates the voltage $[\mathrm{v}]$ at point $p, v_{p} \in[\operatorname{Vmin}, V \max ] ; p \in D$.

It should be recalled that variables $x c c_{p q}$ have been added to the original model presented in [11]. The reason is that they are needed for the proposed heuristics. 


\section{- Objective function}

The objective function (1) minimizes the cost, $Z$, of the initial investment considering: wind and solar generators, regulators, batteries, inverters, cables and meters.

$$
\begin{aligned}
{[M I N] Z=} & \sum_{p \in D} \sum_{a=1}^{A} C A_{a} \cdot x a_{p a}+\sum_{p \in D} \sum_{s=1}^{S} C S_{s} \cdot x S_{p s}+\sum_{p \in D} \sum_{r=1}^{R} C R_{r} \cdot x r S_{p r}+\sum_{p \in D} \sum_{b=1}^{B} C B_{b} \cdot x b_{p b}+\sum_{p \in D} \sum_{i=1}^{I} C I_{i} \cdot x i_{p i}+ \\
& \sum_{p \in D} \sum_{q \in D \backslash\{p\}} \sum_{c=1}^{C} L_{p q} \cdot C C_{c} \cdot x C_{p q c}+\sum_{p \in D} C M \cdot x m_{p}
\end{aligned}
$$

\section{- Constraints}

\section{- Generation and energy storage:}

Constraints (2) and (3) limit the number of wind and solar generators, respectively, and define the generation points. Constraints (4) impose at least one (wind or solar) generator at each generation point. Constraints (5), (6) and (7) are the energy flow, power flow and energy in batteries balances [11, 20]. Constraints (5) impose the conditions of conservation of energy and the satisfaction of requirements of energy demand: at each point, the input energy flow plus the possible energy generated with wind turbines and solar panels must satisfy the energy demand of the point (considering losses) plus the energy demand of the following points of the microgrid. Constraints (6) are analogue to constraints (5) for the conservation and the satisfaction of requirements of power demand. Constraints (7) set the energy to be stored in batteries: for each point, if it is a generation point, the energy in batteries must satisfy the requirements of the point and the following points of the microgrid, considering the autonomy and the admitted discharge level.

$$
\begin{array}{lr}
\sum_{a=1}^{A} x a_{p a} \leq N A \cdot x_{p} & p \in D \\
\sum_{s=1}^{S} x S_{p s} \leq N S \cdot x_{p} & p \in D \\
\sum_{a=1}^{A} x a_{p a}+\sum_{s=1}^{S} x S_{p s} \geq x_{p} & p \in D \\
\sum_{q \in D \backslash\{p\}} f e_{q p}+\sum_{a=1}^{A} E A_{p a} \cdot x a_{p a}+\sum_{s=1}^{S} E S_{p s} \cdot x S_{p s} \geq \frac{E D_{p}}{\eta_{b a t} \eta_{i n v}} \cdot\left(\frac{1}{\eta_{c a b}}+\left(1-\frac{1}{\eta_{c a b}}\right) \cdot x_{p}\right)+\sum_{q \in D \backslash\{p\}} f e_{p q} & p \in D \\
\sum_{q \in D \backslash\{p\}} f p_{q p}+\sum_{i=1}^{I} P I_{i} \cdot x i_{p i} \geq P D_{p} \cdot\left(\frac{1}{\eta_{c a b}}+\left(1-\frac{1}{\eta_{c a b}}\right) \cdot x_{p}\right)+\sum_{q \in D \backslash\{p\}} f p_{p q} & p \in D \\
\sum_{b=1}^{B} E B_{b} \cdot x b_{p b}+\left(\frac{V B}{D B} \cdot \sum_{j \in D} \frac{E D_{j}}{\eta_{b a t} \cdot \eta_{i n v} \cdot \eta_{c a b}}\right) \cdot\left(1-x_{p}\right) \geq \frac{V B}{D B} \cdot\left(\sum_{q \in D \backslash\{p\}} f e_{p q}+E D_{p}\right) & p \in D
\end{array}
$$

\section{- Microgrid:}

Microgrid constraints establish the possible and the maximum energy and power flow [11, 20]. Constraints (8) and (9) relate the energy and power flows respectively to the cable existence: between two points there may be energy or power flow only if there is a cable between them, and the maximum energy or power flow is the demand of all the points considering losses. Constraints (10) determine the radial distribution of the microgrids. Constraints (11) limit the voltage drop between two points connected with a cable of a certain type, which depends on the distance and power flow. Constraints (12) limit the maximum intensity in a cable depending on its type. Constraints (13) establish that two points are connected if there is a cable of any type between them.

$$
\begin{array}{ll}
f e_{p q} \leq\left(\sum_{j \in D} \frac{E D_{j}}{\eta_{b a t} \cdot \eta_{i n v} \cdot \eta_{c a b}}\right) \cdot \sum_{c=1}^{c} x c_{p q c} & p \in D ; q \in D \backslash\{p\} \\
f p_{p q} \leq\left(\sum_{j \in D} \frac{P D_{j}}{\eta_{c a b}}\right) \cdot \sum_{c=1}^{C} x c_{p q c} & p \in D ; q \in D \backslash\{p\} \\
\sum_{q \in D \backslash\{p\}} \sum_{c=1}^{C} x C_{q p c}+x_{p} \leq 1 & p \in D \\
v_{p}-v_{q} \geq \frac{L_{p q} \cdot R C_{c} \cdot f p_{p q}}{V_{n}}-\left(V_{\max }-V_{\min }\right) \cdot\left(1-x C_{p q c}\right) & p \in D ; q \in D \backslash\{p\} ; c=1, \ldots, C
\end{array}
$$




$$
\begin{array}{ll}
\frac{f p_{p q}}{V_{n}}-\left(\sum_{j \in D} \frac{P D_{j}}{V_{\min } \eta_{c a b}}\right) \cdot\left(1-x C_{p q c}\right) \leq I C_{c} & p \in D ; q \in D \backslash\{p\} ; c=1, \ldots, C \\
x C C_{p q}=\sum_{c=1}^{C} x C_{p q c} & p \in D ; q \in D \backslash\{p\}
\end{array}
$$

\section{- Other equipment:}

Constraints (14) define the number of solar regulators depending on the power of the corresponding solar generators. Constraints (15) determine that the inverters can be only placed at generation points. Constraints (16) and (17) establish the placing of electricity consumption meters at the demand points fed by a microgrid.

$$
\begin{array}{ll}
\sum_{r=1}^{R} P R_{r} \cdot x r s_{p r} \geq \sum_{s=1}^{S} P S_{s} \cdot x S_{p s} & p \in D \\
x i_{p i} \leq N I \cdot x_{p} & p \in D ; i=1, \ldots, I \\
\sum_{q \in D \backslash\{p\}} \sum_{c=1}^{C} x C_{p q c} \leq(|D|-1) \cdot x m_{p} & p \in D \\
\sum_{q \in D \backslash\{p\}} \sum_{c=1}^{C} x C_{q p c} \leq x m_{p} & p \in D
\end{array}
$$

\section{MILP-based heuristics}

Three different types of MILP-based heuristic methods stand on the AREMWS model are proposed in the following subsections: Relax and Fix heuristics, Corridor Method heuristics and adhoc heuristics, called Increasing Radius. In common, these algorithms obatin an initial solution by solving a relaxed MILP model. This one, usually infeasible, is used as a starting point to explore a constrained and feasible search space. The differences among the methods rely on the strategies of constraining the search space. In the Relax and Fix heuristics some groups of variables related with the existence of connections of the microgrids are fixed (Section 4.1). In the Corridor Method heuristic the total number of connections is limited (Section 4.2). The Increasing Radius heuristics are developed specifically for this electrification problem in which the fixed connections are those contained in an increasing geographic area (Section 4.3). The following sections explain the three procedures in details.

\subsection{Relax and Fix (RF) heuristics}

Given a MILP model, the keystone of the Fix and Relax methodology introduced by [34] is the following. First, only a reduced subset of (non-fixed) binary variables are kept integer (and the integrity of the other binary variables is relaxed) and the model is solved. Second, the values of some of the (non-relaxed) binary variables are fixed according to the obtained solution. These two steps are repeated until a solution is obtained.

In this subsection, we propose several Relax and Fix (RF) heuristics. The designed RF heuristics have two stages, ST1 and ST2. ST1 consists of solving a relaxed version of AREMWS, considering some integer variables as continuous ones. ST2 starts from the solution obtained from the relaxed model solved in ST1. The solution of ST1, obviously, is not necessarily a feasible solution but is useful in order to obtain clues about how a good solution looks like. In that sense, in ST2 the values of some variables are fixed (from the non-relaxed variables of the solution obtained in ST1) and then AREMWS is solved. The solution returned by the RF heuristics is the output of ST2. The proposed RF heuristics differ exclusively in the variables to relax in the first stage (ST1) and / or the variables to be fixed in the second stage (ST2).

\subsubsection{General scheme of the RF heuristics}

Formally detailed, the stages of the RF heuristics are:

1. Let $X=\left\{x_{1}, \mathrm{x}_{2}, \ldots, \mathrm{x}_{N}\right\}$ be the set of integer (including binary) variables of AREMWS, where $N=|X|$ and $x_{i}$ $(i=1, \ldots, \mathrm{N})$ are the number of integer variables and the $i^{\text {th }}$ integer variable, respectively. 
2. Let $I R=\left\{r_{1}, r_{2}, \ldots, r_{N_{1}}\right\} \subseteq\{1, \ldots, N\}$ be the set of indexes of the integer variables to relax, where $N_{1}=|I R|$ and $x_{r}(r \in I R)$ are the number of variables and the $r^{\text {th }}$ variable to relax, respectively.

3. Solve AREMWS relaxing the integrity of variables $x_{r}(r \in I R)$ : Let $\bar{x}_{i} \quad(i=1, \ldots, \mathrm{N})$ be the value of variable $x_{i}$ of the obtained solution.

ST2:

4. Let $I F=\left\{f_{1}, f_{2}, \ldots, f_{N_{2}}\right\} \subseteq\{1, \ldots, N\} \backslash I R$ be the set of indexes of variables to fix, where $N_{2}=|I F|$ and $x_{f}$ ( $f \in I F$ ) are the number of variables and the $f^{\text {th }}$ variable to fix, respectively.

5. Fix the values of variables $x_{f}$ to the values obtained at step 3; i.e., $x_{f}=\bar{x}_{f}(f \in I F)$.

6. Solve AREMWS (without relaxing any variable) and return the obtained solution.

\subsubsection{Variables to relax}

In order to define the variables to relax a preliminary computational experiment was carried out. The variables that represent the number of wind turbines of type $a$ at point $p\left(x a_{p a}\right)$ and photovoltaic panels of type $s$ at point $p\left(x s_{p s}\right)$ are not appropriate candidates to relax. The reason is that, due to economies of scale and the relaxation of these variables, the solutions obtained in stage ST1 tend strongly to have no microgrids and to place fractions of the most powerful generators (since they have the lowest cost/power ratio). Thus, these solutions would not be useful to fix variable values in stage ST2, as they are not a good reference of which microgrids are part of a good solution. On the other hand, due to an opposite phenomenon, to relax all variables related with the existence of cable $\left(x c c_{p q}\right.$ and $\left.x c_{p q c}\right)$ would tend in stage ST1 to excessively extend the microgrids placing fractions of different type of cables.

In order to define the set of candidate variables to relax (CVR) in stage ST1 were considered those that relate to the number of batteries type $b$ at point $p\left(x b_{p b}\right)$, the number of inverters of type $i$ at point $p\left(x i_{p i}\right)$, the number of regulators of type $r$ at point $p$ in $\left(x r s_{p r}\right)$, the existence of the cable of type $c$ from point $p$ to point $q\left(x c_{p q c}\right)$ and the placing of electric meter at point $p\left(x m_{p}\right)$. Four relaxation options are proposed (R1-R4) in Table 1. Other combinations of relaxations with the CVR were tested but discarded by the preliminary experiment.

Table 1. 4 relaxation options. The symbol $\checkmark(\mathbf{x})$ indicates that the variables are (not) relaxed.

\begin{tabular}{lccccc} 
Relaxation & $x b_{p b}$ & $x i_{p i}$ & $x r s_{p r}$ & $x c_{p q c}$ & $x m_{p}$ \\
option & & & & & \\
\hline R1 & $\checkmark$ & $\checkmark$ & $\checkmark$ & $\checkmark$ & $\checkmark$ \\
R2 & $\checkmark$ & $\checkmark$ & $\checkmark$ & $\mathbf{x}$ & $\checkmark$ \\
R3 & $\times$ & $\checkmark$ & $\checkmark$ & $\checkmark$ & $\checkmark$ \\
R4 & $\checkmark$ & $\mathbf{x}$ & $\checkmark$ & $\checkmark$ & $\checkmark$ \\
\hline
\end{tabular}

\subsubsection{Variables to fix}

Regarding variables to fix in stage ST2, six fixation options (F1 - F6) are proposed over the variables of existence of connection from point $p$ to point $q, x c c_{p q}$, or the variables of existence of generators at point $p\left(x_{p}\right)$. Let $\overline{x C C}_{p q}$ and $\bar{x}_{p}$ be the values of variables $x c c_{p q}$ and $x_{p}$ of the solution obtained in stage ST1, respectively. The different options are next described:

F1. The existence of connection between two points is fixed (the absence is not fixed). That is, $x c c_{p q}=1$ $\left(p \in D ; q \in D \backslash\{p\}: \overline{x C C}_{p q}=1\right)$.

F2. The absence of connection between two points is fixed (the existence is not fixed). That is, $x c c_{p q}=0$ $\left(p \in D ; q \in D \backslash\{p\}: \overline{x C C}_{p q}=0\right)$.

F3. The existence and absence of connection between two points are fixed. That is, $x c c_{p q}=\overline{x C C}_{p q}$ $(p \in D ; q \in D \backslash\{p\})$.

F4. The existence of generators at a point is fixed (the absence is not fixed). That is, $x_{p}=1\left(p \in D: \bar{x}_{p}=1\right)$. 
F5. The absence of generators at a point is fixed (the existence is not fixed). That is, $x_{p}=0 \quad\left(p \in D: \bar{x}_{p}=0\right)$.

F6. The existence and absence of generators at a point are fixed. That is, $x_{p}=\bar{x}_{p}(p \in D)$.

Combinations between F1, F2 and F3 with F4, F5 and F6 were not considered as the search space to explore is reduced (therefore, worst solutions may be obtained) and options F1 to F6 require low computational time (results are shown in Section 5).

\subsubsection{Proposed RF heuristics}

In total, twenty four heuristics of relax and fix are proposed which arise from combining the four options of variables to relax in stage ST1 (R1-R4) and the six options of variables to fix in stage ST2 (F1-F6). Let $R F a b(a=1, \ldots, 4 ; b=1, \ldots, 6)$ be the heuristic with the relaxation option $R a$ and the fixation option $F b$. Finally, we introduce the parameters $T_{1}^{R F}$ and $T_{2}^{R F}$ as the maximum resolution times in stages ST1 and ST2, respectively.

\subsection{Heuristics based on Corridor Method (BCM)}

The general concept of Corridor Method (CM) has been presented as a hybrid metaheuristic, linking together mathematical programming with heuristics [35, 36]. CM is based on the use of an exact method applied to a subspace of the solution space of a specific problem. Given a hard optimization problem, let x be the feasible space and $M$ be an exact method (in this paper AREMWS) that is inefficient for large instances of the problem but efficient for smaller ones.

CM defines a neighborhood, or corridor, built in consideration of the method $M$ used to explore it: let $\bar{x} \in \mathrm{x}$ be a current solution, from which CM builds a neighborhood, $N(\bar{x})$, that can be effectively explored by the method $M . N(\bar{x})$ should be reasonably large and built in such a way that it could be explored in (pseudo) polynomial time using method $M$ [37]. Typically, the corridor $N(\bar{x})$ around a current solution is defined by imposing exogenous constraints on the original problem to identify a limited portion of the search space [38].

Thus, as second proposal, MILP heuristics based on Corridor Method (BCM) are developed. All those heuristics are based on search iteratively in the neighborhood of the current solution.

\subsubsection{The definition of the neighborhood}

The constraints proposed for the implementation of $N(\bar{x})$ are as follows:

- The number of connection and disconnection changes between the current solution and a neighbor is limited to a value $L$, as formulated in (18) and (19), respectively:

$$
\begin{aligned}
& \sum_{p \in D} \sum_{\substack{q \in D \backslash\{p\}: \\
\overline{x C C_{p q}}=0}} x C C_{p q} \leq L \\
& \sum_{p \in D} \sum_{\substack{q \in D \backslash\{p\}: \\
\overline{x c c_{p q}}=1}}\left(1-x c C_{p q}\right) \leq L
\end{aligned}
$$

- It is not allowed to change the current flow directions:

$$
x C C_{q p}=0 \quad p \in D ; q \in D \backslash\{p\}: \overline{x C C}_{p q}=1
$$

- Connections between two points that are not connected at any microgrid in the current solution are not allowed, where $N C=\left\{p \in D: \underset{q \in D \backslash\{p\}}{\forall} \overline{x C C}_{p q}+\overline{x C C}_{q p}=0\right\}$ :

$$
x C C_{p q}=0 \quad p \in N C ; q \in N C \backslash\{p\}
$$


- Connections between two points $p$ and $q$ in which the distance between them is larger than their break even distance, $B E D_{p q}$, are not allowed. $B E D_{p q}$ is the distance with a connection cost equal to the maximum cost of electrifying individually points $p$ or $q$. Thus, $B E D_{p q}$ is an estimation of the break even distance that may worth to connect both points instead of electrifying one of them individually:

$$
x C c_{p q}=0 \quad p \in D ; q \in D \backslash\{p\}: L_{p q}>B E D_{p q}
$$

Constraints (18) and (19) define the corridor around the number of connection and disconnection changes. A preliminary computational experiment showed that these constraints do not reduce the solution space enough to be explored efficiently. Thus, we propose the additional constraints (20) - (22) in order to obtain a small enough neighborhood that can be explored within a practical time.

\subsubsection{General scheme of the BCM heuristics}

The pseudo-code of the developed BCM heuristics is presented above:

1: Apply ST1 of the RF heuristics (see Section 4.1.1) and let $\bar{x}$ be the obtained solution.

2: Local search process:

While (not stopping criterion) do

Build the neighborhood $N(\bar{x})$ by imposing constraints (18) to (22) to AREMWS.

Solve AREMWS (without relaxing any variable) and return the new current solution, $\bar{x}$. end while

Stopping criterion occurs when the current solution does not improve or a time limit is attained.

\subsubsection{Proposed BCM heuristics}

Four BCM heuristics are proposed as a result of the relaxation options R1 to R4, introduced in Section 4.1.2, used to achieve a first solution in order to start the local search process. We denote by BCM $a$ as the heuristic with relax option $\mathrm{R} a(a=1, \ldots, 4)$. Finally, the parameters $T_{1}^{B C M}$ and $T_{2}^{B C M}$ are defined as the maximum resolution time at steps 1 (initial solution) and 2 (local search) of the algorithm, respectively.

\subsection{Increasing Radius (IR) heuristics}

The idea behind the iterative heuristics called Increasing Radius (IR) is shown as follows. At each iteration $i$, point $p_{i}$ $\left(p_{i} \in D\right)$ is selected and, starting from this point, a solution is constructed. The heuristics end when all demands points have been selected as starting points or a maximum computational time is achieved and the solution found with the least cost is returned.

To determine the point $p_{i}$, we use Grid Generation Score (GGS) [29] defined as an index which evaluates outstanding resource potential (renewable energy potential in a specific site) in comparison with the surrounding points and the energy demand concentration around the point. Thus, we choose the demand point with the $i$-th highest GGS value.

The process to construct a solution (starting from point $p_{i}$ ) is also iterative and it is as follows. In the sequence, at each iteration $j$ of the solution construction process, the surface of a sphere with centre at $p_{i}$ and radius $R_{i j}$ becomes the limit that separates all the demand points in two regions: interior region of non-relaxation, $I N T_{i j}$ $\left(I N T_{i j}=\left\{p \in D \mid L_{p, p_{i}} \leq R_{i j}\right\}\right)$ and exterior region of relaxation, $E X T_{i j}\left(E X T_{i j}=D \backslash I N T_{i j}\right)$. Figure 2a ilustrates an example of the first iteration of a solution construction process, in which the small points represent the demand points, the grey circle is region $I N T_{i 1}$ around point $p_{i}$ and the darkest area is region $E X T_{i j}$. Iteratively, the radius of the sphere increases (from now on, expansion) until all the points of the instance are incorporated (i.e, the exterior region is void). At iteration $j$, AREMWS is solved with some variables relaxed and other variables fixed. The variables to relax are some of those associated to the points of the exterior region $\left(E X T_{i j}\right)$. The variables to fix are some of associated to the 
points of the interior region of the previous iteration $\left(I N T_{i, j-1}\right.$, where $\left.I N T_{i 0}=\varnothing\right)$, and they are fixed with the values of the solution obtained with AREMWS at the previous iteration (i.e, iteration $j-1$ ). The increase of the radius at each iteration $j$ is determined by a parameter that indicates the number of points $\left(n p_{0}\right)$ that are wanted to be added at each expansion. Thus, the expansion is determined by the incorporation of new $n p_{0}$ points rather than a prefixed increment of the radius. Figure $2 \mathrm{~b}$ illustrates an example of the second iteration of the solution construction process, in which the white circle is the "fixed" area. Note that $n p_{0}$ is equal to 7 in the example shown in Figure 2.

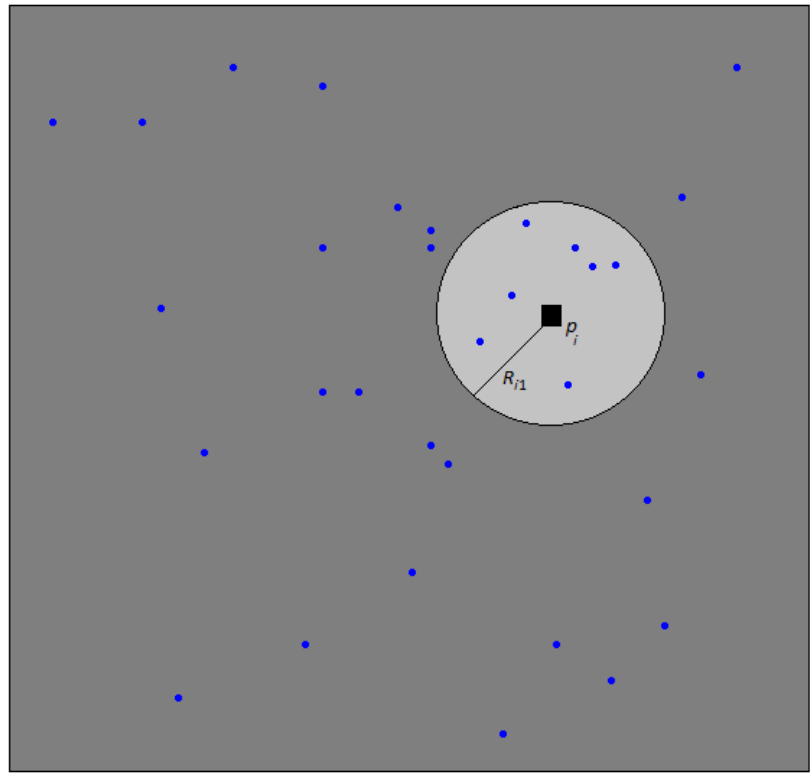

Figure 2a. Solution construction: first iteration $(j=1)$

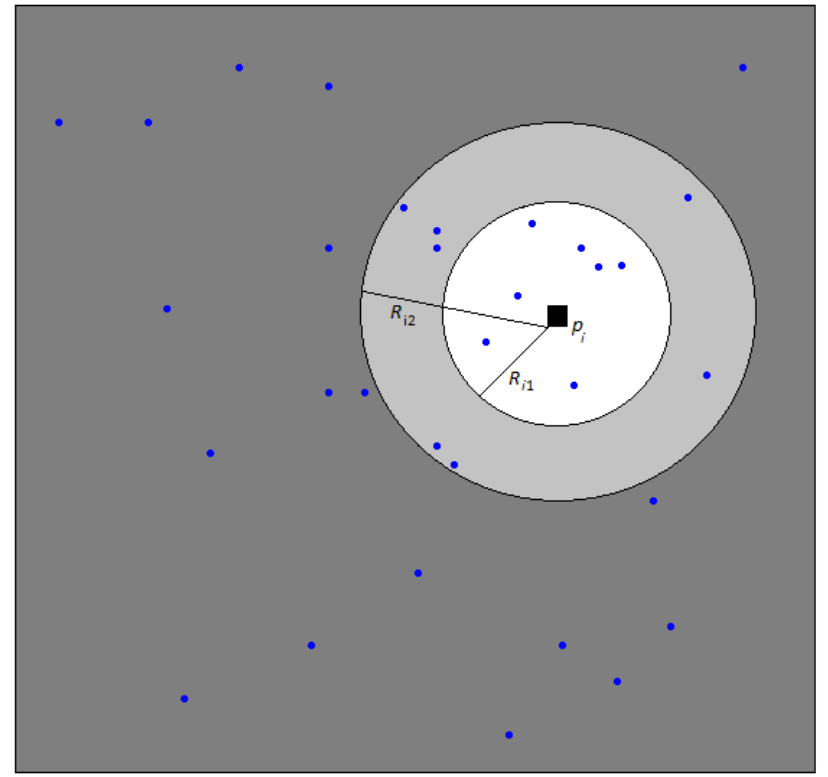

Figure 2b. Solution construction: second iteration $(j=2)$

\subsubsection{General scheme of the IR heuristics}

Recall that $X$ is the set of integer (including binary) variables of AREMWS ( $x \in X$ ) and $N=|X|$ (see Section 4.1.1).

Let $\operatorname{IREXT}_{i j}=\left\{r_{1 i j}, \mathrm{r}_{2 i j}, \ldots, \mathrm{r}_{N_{1 i j}^{I R}}\right\} \subseteq\{1, \ldots, N\}$ be the set of indexes of the variables to relax associated to exterior points $p \in E X T_{i j}$, where $N_{1 i j}^{I R}=\left|I R E X T_{i j}\right|$ and let $x_{r}\left(r \in I R E X T_{i j}\right)$ be the number of variables to relax and the $r^{\text {th }}$ variable to relax, respectively.

Let $I F_{i j}^{I R}=\left\{f_{1 i j}, f_{2 i j}, \ldots, f_{N_{2 i j}^{I R}}\right\} \subseteq\{1, \ldots, N\} \backslash I R E X T_{i j}$ be the set of indexes of variables to fix associated to interior points $p \in I N T_{i j}$, where $N_{2 i j}^{I R}=\left|I F_{i j}^{I R}\right|$ and let $x_{f}\left(f \in I F_{i j}^{I R}\right)$ be the number of variables to fix and the $f^{\text {th }}$ variable to fix, respectively.

The algorithm of the Increasing Radius heuristics is the following:

$i=1$

While ( $i \leq|D|$ or time limit is attained) do:

$p_{i}=$ Demand point with the $i$-th highest GGS value

$n p=0 ; j=1$

While $(n p<|D|)$ do:

$n p=\min \left(n p+n p_{0},|D|\right)$ and let $R_{i j}$ be the minimum value so $\left|I N T_{i j}\right|=n p$

Fix the values of variables $x_{f}: x_{f}=\bar{x}_{f}\left(f \in I F_{i, j-1}^{I R}\right)$

Relax variables $x_{r} \in I R E X T_{i j}$

Solve the relaxed AREMWS and obtain the values $\bar{x}_{f}\left(f \in I F_{i j}^{I R}\right)$

$j=j+1$ 
End while

$i=i+1$

End while

Return the least cost solution.

\subsubsection{Variables to relax}

In order to define the set of variables to relax in the IR heuristics, the relaxing options (R1-R4) in the RF heuristics (Section 4.1.2) are also considered.

\subsubsection{Variables to fix}

In order to define the set of variables to fix in the IR heuristics, the fixing options (F1-F6) in the RF heuristics (Section 4.1.3) are also considered.

\subsubsection{Proposed heuristics}

Twenty four variants IR heuristics are proposed, arising from combining the four options of relaxation and the six options of fixation. Moreover, we define $\operatorname{IRab}(a=1, \ldots, 4 ; b=1, \ldots, 6)$ as the heuristic with the relaxation option $R a$ and the fixation option $F b$.

The total maximum resolution time is $T$ and the maximum time to construct solution $i$ (let that time be called $T C_{i}$ ) is all the remaining time (i.e, $T C_{i}$ is $T$ minus the time spent to construct the previous solutions). The maximum time to solve the relaxed AREWMS at each iteration $j$ of the construction of solution $i$ is the remaining time divided by $\left\lceil|D| /\left(j \cdot n p_{0}\right)\right\rceil$ (i.e, divided by the number of the remaining expansions in the construction of solution $i$.

\section{Computational experiment}

The instances of the computational experiment were randomly generated based on the characteristics of a real project in the Peruvian community of El Alumbre [8], in the area of $3.5 \times 3.5 \mathrm{~km}^{2}$ in which the wind map was made. The wind resource map and the data of the energy generated with each type of turbine were calculated using WAsP [39].

The main data of the electrical equipment considered in the project are summarized in Table 2.

Table 2. Electrical equipment considered in the project.

\begin{tabular}{|l|l|l|l|}
\hline Equipment & Types & Cost & Other characteristics \\
\hline Wind turbines & 4 & from $\$ 1394$ to $\$ 8732$ & $\begin{array}{l}\text { energy generation: calculated using WAsP [39], up to } \\
16500 \mathrm{Wh} / \mathrm{day} \\
\text { maximum power: from } 300 \mathrm{~W} \text { to } 3500 \mathrm{~W}\end{array}$ \\
\hline Solar panels & 4 & from $\$ 451$ to $\$ 1000$ & $\begin{array}{l}\text { energy generation: from } 217 \mathrm{Wh} / \mathrm{day} \text { to } 651 \mathrm{Wh} / \mathrm{day} \\
\text { maximum power: from } 50 \mathrm{~W} \text { to } 150 \mathrm{~W}\end{array}$ \\
\hline Solar regulators & 4 & from $\$ 67$ to $\$ 125$ & maximum power: from $50 \mathrm{~W}$ to $200 \mathrm{~W}$ \\
\hline Batteries & 4 & from $\$ 225$ to $\$ 325$ & capacity: from $1500 \mathrm{Wh}$ to $3000 \mathrm{Wh}$; efficiency: 0.85 \\
\hline Inverters & 4 & from $\$ 377$ to $\$ 2700$ & maximum power: from $300 \mathrm{~W}$ to $3000 \mathrm{~W}$; efficiency: 0.85 \\
\hline Cables & 3 & $\$ 5.9 / \mathrm{m}$ and $\$ 6.3 / \mathrm{m}$ & resistance: $2.71 \Omega / \mathrm{km}$ and $1.7 \Omega / \mathrm{km}$ \\
\hline
\end{tabular}

Based on these characteristics, the set of testing instances was generated considering the different scenarios summarized in Table 3.

Table 3. Scenarios considered in the set of instances generation.

\begin{tabular}{|l|l|l|}
\hline Aspect & Options & Characteristics \\
\hline Number of demand points & 10 & $10,20,30,40,50,60,70,80,90,100$. \\
\hline Density & 2 & $\begin{array}{l}\text { 1) } 25 \% \text { of demand points concentrated in 20\% of the community area } \\
\text { 2) } 50 \% \text { of demand points concentrated in } 20 \% \text { of the community area. The } \\
\text { exact locations of the points were randomly generated (observing the } \\
\text { densities of the scenarios) }\end{array}$ \\
\hline Demand (energy/power) per & 2 & 1) $280 \mathrm{Wh} /$ day (200 W) \\
\hline
\end{tabular}




\begin{tabular}{|l|l|l|}
\hline house & & 2) $420 \mathrm{Wh} /$ day (300 W) \\
\hline Wind potential & 2 & $\begin{array}{l}\text { 1) normal (according to the wind map) } \\
\text { 2) high (multiplying by } 1.5 \text { the values of the wind map) }\end{array}$ \\
\hline
\end{tabular}

For each combination of scenarios (number of demand points, density, demand and wind potential), 5 instances were generated, giving a total of $10 \times 2 \times 2 \times 2 \times 5=400$ instances.

The tests were carried out in a PC $3.16 \mathrm{GHz}$ Intel Core 2 Duo E8500 with 3.46 GB of RAM. The algorithms were coded in $\mathrm{C}++$ and the MILP models were solved with IBM ILOG CPLEX Optimizer 12.2. The time limit for all heuristics is set to 3600 seconds.

The proposed heuristics were first compared with a starighforward MILP-based heuristic, called henceforth AREMWS1h, that consists in limiting AREMWS to 3600 seconds. Let this heuristic be called AREMWS-1h. Thirty two instances with 10 demand points are solved with AREMWS-1h before 3600 seconds (i.e, proven optimal solutions are achieved). For the remaining instances, no (proven) optimal solutions are obtained. Results achieved with the RF, BCM and IR heuristics and their parameter values are reported in Section 5.1, 5.2 and 5.3, respectively. In Section 5.4, the proposed heuristics are compared among them. All results are summarized in Tables 4, 5 and 6, where AI\% and MI\% are the average and maximum improvement (in \%) with respect to the results of the heuristic AREMWS-1h, respectively.

The statistical significance of the results of the computational experiment was evaluated by the Paired t-test, which tests the mean difference between paired observations; with a significance level of 5\%, using the Minitab 15.1 Statistical Software.

\subsection{Results of the RF heuristics}

The computational time for the RF heuristics is divided as follows: $T_{1}^{R F}$ is set to 2700 seconds and $T_{2}^{R F}$ is set to the remaining computational time (900 seconds). The reason is that a preliminary experiment showed that ST2 is quicker than ST1. Additionally, in order to accelerate ST1, the relative gap between the value of the cost function and its lower bound at this stage was limited to $25 \%$ for instances of 10 and 20 points. For instances bigger than 20 points (80\% of total instances) a gap limit equal to $25 \%$ was not active in practice because computational time of ST1 run out with gaps bigger than 25\%. Experiments made with greater gap limits (50\% and 75\%) showed worse results for all instances.

The best results were obtained with RF12, RF13 and RF14 heuristics, which are presented in Table 4. As it can be seen, the three best RF heuristics obtain, on average, better solutions than AREMWS-1h, and the statistical test confirmed the differences with AREMWS-1h are significant. The average values of AI\% obtained with RF14, RF12 and RF13 are 1.42, 1.19 and 0.42, respectively. Those heuristics give similar results to AREMWS-1h for instances up to 30 demand points. On the other hand, they obtain significantly better results than AREMWS-1h for larger instances, and especially for instances from 60 to 80 demand points. All three best RF heuristics relax variables $x b_{p b}, x i_{p i}, x r s_{p r}, x c_{p q c}$ and $x m_{p}$ in their first stage ST1 and they differ in the second stage ST2. In particular, RF14, which is the best RF heuristic, fix only the existence of generators, with cost decreases up to 16\% with respect to AREMWS-1h.

\begin{tabular}{c|c|c|c|c|c|c} 
Table 4. 3 best results obtained with RF heuristics (compared with AREMWS-1h) \\
\hline \multirow{2}{*}{$|\boldsymbol{D}|$} & \multicolumn{2}{|c}{ RF12 } & \multicolumn{2}{c}{ RF13 } & \multicolumn{2}{c}{ RF14 } \\
\cline { 2 - 7 } & AI\% & MI\% & AI\% & MI\% & AI\% & MI\% \\
\hline 10 & -0.21 & 0.06 & -0.41 & 0.08 & -0.20 & 0.19 \\
20 & -1.15 & 0.74 & -1.37 & 0.74 & -0.96 & 0.74 \\
30 & -0.62 & 3.24 & -1.71 & 2.57 & -0.71 & 3.11 \\
40 & -1.87 & 6.11 & -2.17 & 12.58 & 0.48 & 9.75 \\
50 & 0.83 & 7.50 & -0.41 & 7.95 & 2.54 & 10.50 \\
60 & 3.98 & 13.58 & 2.26 & 12.83 & 4.70 & 12.40 \\
70 & 4.52 & 12.70 & 3.28 & 10.75 & 3.70 & 16.14 \\
80 & 4.09 & 14.25 & 2.63 & 12.05 & 2.24 & 12.66 \\
90 & 1.49 & 7.41 & 1.02 & 5.41 & 1.65 & 10.72 \\
100 & 0.80 & 1.86 & 1.10 & 7.34 & 0.74 & 1.86 \\
\hline All & 1.19 & 14.25 & 0.42 & 12.83 & 1.42 & 16.14 \\
\hline
\end{tabular}


Similarly to the RF heuristics, the second step of the BCM heuristics (local search) is quicker than the first step (calculation of the initial solution). Thus, $T_{1}^{B C M}$ is set to 2700 seconds and $T_{2}^{B C M}$ is set to 900 seconds. Regarding parameter $L$ (limit of connection and disconnection changes), 3 values were tested in each BCM heuristic: $L \in\{1,2,5\}$.

The best results were obtained with BCM1 with $L=1,2$ and 5, which are presented in Table 5 . Their average values of AI\% 0.25, 0.35 and 1.66, respectively. Therefore, the three best BCM heuristics obtain better solutions than AREMWS$1 \mathrm{~h}$, and the statistical test confirmed the differences are significant. As it can be seen, from 10 to 40 and for 100 demand points, AI\% presents values lower than or near to 0, so AREMWS-1h performs better than or similar to the shown BCM heuristics. However, from 50 to 90 demand points a significant improvement of the BCM results is observed with AI\% from around $1 \%$ to $6 \%$. The best results are obtained with BCM1 heuristic and $L=5$ (that means relaxing option R1 and limit of connection and disconnection changes fixed at 5), with cost decreases up to $15 \%$.

Table 5. 3 best results obtained with BCM heuristics (compared with AREMWS-1h).

\begin{tabular}{c|c|c|c|c|c|c}
\hline \multirow{2}{*}{$|\boldsymbol{D}|$} & \multicolumn{2}{|c|}{ BCM1 $(\boldsymbol{L}=\mathbf{1})$} & \multicolumn{2}{c|}{ BCM1 $(\boldsymbol{L}=\mathbf{2})$} & \multicolumn{2}{c}{ BCM1 $(\boldsymbol{L}=\mathbf{5})$} \\
\cline { 2 - 7 } & $\mathbf{A I \%}$ & $\mathbf{M I \%}$ & $\mathbf{A I \%}$ & $\mathbf{M I} \%$ & $\mathbf{A I \%}$ & $\mathbf{M I} \%$ \\
\hline 10 & -0.86 & 0 & -0.65 & 0.19 & -0.51 & 0.06 \\
20 & -1.65 & 0.77 & -1.38 & 0.73 & -0.46 & 0.83 \\
30 & -2.75 & 0.59 & -2.57 & 0.64 & -0.93 & 2.89 \\
40 & -3.27 & 2.54 & -3.12 & 7.27 & -1.15 & 8.76 \\
50 & -0.13 & 9.92 & 0.26 & 8.18 & 1.74 & 11.57 \\
60 & 2.58 & 8.37 & 2.48 & 11.28 & 4.42 & 15.22 \\
70 & 4.16 & 13.80 & 3.94 & 12.43 & 6.08 & 15.08 \\
80 & 3.99 & 15.76 & 3.58 & 14.17 & 5.46 & 15.67 \\
90 & 0.34 & 5.18 & 0.78 & 7.14 & 1.15 & 9.85 \\
100 & 0.09 & 2.33 & 0.14 & 2.34 & 0.76 & 11.58 \\
\hline All & 0.25 & 15.76 & 0.35 & 14.17 & 1.66 & 15.67 \\
\hline
\end{tabular}

\subsection{Results for the IR heuristics}

In this study, we take $n p_{0} \in\{5,10,20,30,40,50,60\}$.

The best relax and fix options are R1 and F2, respectively, since the best results, shown in Table 6, were obtained with IR12 setting $n p_{0}$ to 40,50 or 60 . The obtained average values of AI\% are 3.00, 3.36 and 2.83, respectively. Therefore, the three best IR heuristics obtain better solutions than AREMWS- $1 \mathrm{~h}$, and the statistical test confirmed the differences are significant. Note that the values of $\mathrm{AI} \%$ up to $n p_{0}$ demand points is 0 ; the reason is that, in that case, the IR heuristics are equivalent to AREMWS-1h. From 60 to 100 demand points, a significant improvement of AI\% is observed, specially when $n p_{0}=50$ : on average, from almost $5 \%$ to almost $8 \%$. Moreover, when $n p_{0}=50$, the maximum improvement MI\% is up to $20.31 \%$

Table 6. 3 best results obtained with IR heuristics (compared with AREMWS-1h).

\begin{tabular}{|c|c|c|c|c|c|c|}
\hline \multirow{2}{*}{$|D|$} & \multicolumn{2}{|c|}{$\operatorname{IR12}\left(n p_{0}=40\right)$} & \multicolumn{2}{|c|}{$\operatorname{IR12}\left(n p_{0}=50\right)$} & \multicolumn{2}{|c|}{$\operatorname{IR12}\left(n p_{0}=60\right)$} \\
\hline & AI\% & MI\% & AI\% & MI\% & AI\% & MI\% \\
\hline 10 & 0 & 0 & 0 & 0 & 0 & 0 \\
\hline 20 & 0 & 0 & 0 & 0 & 0 & 0 \\
\hline 30 & 0 & 0 & 0 & 0 & 0 & 0 \\
\hline 40 & 0 & 0 & 0 & 0 & 0 & 0 \\
\hline 50 & 1.08 & 9.30 & 0 & 0 & 0 & 0 \\
\hline 60 & 4.91 & 13.14 & 4.99 & 10.73 & 0 & 0 \\
\hline 70 & 6.35 & 15.93 & 6.20 & 20.31 & 6.05 & 17.39 \\
\hline 80 & 7.26 & 17.06 & 7.34 & 19.54 & 7.09 & 19.11 \\
\hline 90 & 4.85 & 12.11 & 7.11 & 19.28 & 7.57 & 18.77 \\
\hline 100 & 5.54 & 15.07 & 7.93 & 18.28 & 7.59 & 22.98 \\
\hline All & 3.00 & 17.06 & 3.36 & 20.31 & 2.83 & 22.98 \\
\hline
\end{tabular}




\subsection{Comparison of the three types of heuristics}

On average, the best results are obtained with an IR heuristic. Specifically, IR12 with $n p_{0}=50$ has an average AI\% equal to 3.36.The statistical test confirmed IR12 with $n p_{0}=50$ obtain the best results and the differences with the other heuristics are significant.

Complementary, as results vary depending on the number of points of the instances, we would suggest the use of different heuristics depending on the size of the instance to solve, as summarized in Table 7.

- From 10 to 30 and from 60 to 100 demand points. The best option is to apply IR12 with different values of parameter $n p_{0}$, whose value can be easily introduced by the user.

- $\quad$ From 40 to 50 demand points. The best option is to apply RF14.

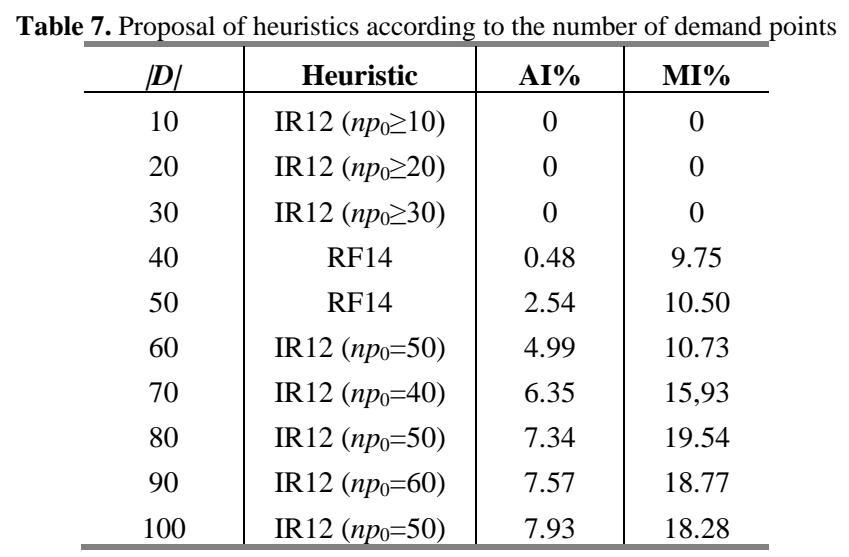

\section{Conclusions}

This paper presents the problem of rural electrification project using wind and solar generation and the distribution of electricity by individual and microgrid systems. In order to solve the problem we design 3 type of heuristic methods based on a MILP model: Relax and Fix heuristics, heuristics based on Corridor Method and adhoc here named Increasing Radius heuristics. In all heuristics first the relaxed model is applied to obtain a base solution which is useful for reduce the search space. However, different strategies to reduce this space are used: in the Relax and Fix heuristics some groups of variables related with connections and/or generators in the microgrids are fixed; in the Corridor Method heuristics the number of total connections is limited, among other additional constraints; and in the Increasing Radius heuristics the connections and/or generators contained in an iteratively increasing geographic area are fixed. For each type of heuristic several options are developed and tested.

An extensive computational experiment is carried out with generated instances based on a real project and the main results of the different heuristics are showed and compared. First the best relaxation and fixation options within each type of algorithm is detected. Then the 3 types of heuristics are compared and concluded that the best heuristic to apply depends on the number of demand points of the instance to solve: for medium size instances we propose to use a Relax and Fix heuristic and for small and large instances we suggest to use Increasing Radius heuristics.

\section{References}

[1] IEA 2013. www.iea.org/topics/energypoverty, visited in $22^{\text {th }}$ May 2015

[2] El Bassam, N. (2001) Renewable energy for rural communities. Renewable Energy, 24, 401-408.

[3] Chaurey, A., Ranganathana, M., \& Mohanty, P. (2004) Electricity access for geographically disadvantaged rural communities-technology and policy insights. Energy Policy, 32, 1693-1705.

[4] Lew, D. J. (2000) Alternatives to coal and candles: wind power in China. Energy Policy, 28, 271-286. 
[5] Ferrer-Martí, L., Garwood, A., Chiroque, J., Ramirez, B., Marcelo, O., Garfi, M., \& Velo, E, (2012). Evaluating and comparing three community small-scale wind electrification projects. Renewable and Sustainable Energy Reviews, 16 , 5379-5390.

[6] Nandi, S. K., \& Gosh, H. R. (2010) Prospect of wind-PV-battery hybrid power system as an alternative to grid extension in Bangladesh. Energy, 35, 3040-3047.

[7] Zhou, W., Lou, C., Li, Z., Lu, L., \& Yang, H. (2010) Current status of research on optimum sizing of stand-alone hybrid sola-wind power generation systems. Applied Energy, 87, 380-389.

[8] Ferrer-Martí, L., Garwood, A., Chiroque, J., Escobar, R., Coello, J., \& Castro, M. A. (2010) A community smallscale wind generation Project in Peru. Wind Engineering, 34, 277-288.

[9] Camblong, H., Sarr, J., Niang, A. T., Curea, O., Alzola, J. A., Sylla, E. H., \& Santos, M. (2009) Micro-grids project, Part 1: Analysis of rural electrification with high content of renewable energy sources in Senegal. Renewable Energy, 34, 2141-2150.

[10] Kirubi. C., Jacobson, A., Kamme, D. M, \& Mills, A, (2009) Community-based electric micro-grids can contribute to rural development: Evidence from Kenya. World Development, 37, 1208-1221.

[11] Ferrer-Martí, L., Domenech, B., García-Villoria, A., \& Pastor, R. (2013) A MILP model to design hybrid windphotovoltaic isolated rural electrification projects in developing countries. European Journal of Operational Research, 226, 293-300.

[12] Georgopoulou, E., Lalas, D., \& Papagiannakis, L. (1997) A multicriteria decision aid approach for energy planning problems. The case of renewable energy option. European Journal of Operational Research, 103, 38-54.

[13] Luna-Rubio, R., Trejo-Perea, M., Vargas-Vázquez, D., \& Rios-Moreno, G. J. (2012) Optimal sizing of renewable hybrids energy sistems. A review of methodologies. Solar Energy, 86, 1077-1088.

[14] Henao, F., Cherni, J. A., Jaramillo, P., \& Dyner, I. (2012) A multicriteria approach to sustainable energy supply for the rural poor. European Journal of Operational Research, 218, 801-809.

[15] Rojas-Zerpa, J. C., \& Yusta, J. M. (2014) Methodologies, technologies and applications for electric supply planning in rural remote areas. Energy for sustainable development, 20, 66-76.

[16] Thirault, D., Bésanger, Y., Hadjsaid, N., Almeida, A., \& Huard, G. (2002) Cost Estimation of Different Electric Systems for Rural Areas in Developing Countries. Power Engineering Society Summer Meeting IEEE, 1, 23-28.

[17] Keller, S., Naciri, S., Nejmi, A., \& Dos Ghali, J. (2007) Simulation-based decision support tool for electrification of isolated areas using a network with multiple renewable sources. International Conference on Clean Electrical Power, $1-8$.

[18] Michelon, B., Nejmi, A., Dos Ghali, J., Dahman, A., \& Bolay, J. (2008) Electrification of Isolated Areas by Interconnecting Renewable Sources (ERD Project): Lessons Learned. Proceeding of the International Conference on Sustainable Energy Technologies, 747-752.

[19] Lambert, T.W., \& Hittle, D. C. (2000) Optimization of autonomous village electrification systems by simulated annealing. Solar Energy, 68, 121-32.

[20] Ferrer-Martí, L, Pastor, R., Capó, G. M., \& Velo, E. (2011) Optimizing microwind rural electrification projects. A case study in Peru. Journal of Global Optimization, 50, 127-143.

[21] Baños, R., Manzano-Agugliaro, F., Montoya, F. G., Gil, C, Alcayde. A., \& Gómez, J. (2011) Optimization methods applied to renewable and sustainable energy: A review. Renewable \& Sustainable Energy Reviews 15, 175366.

[22] White, L, Smith, H., \& Currie, C. (2013) OR in developing countries: A review. European Journal of Operational Research, 226, 293-300.

[23] Kanase-Patil, A.B., Saini, R. P, \& Sharma, M. P. (2010) Integrated renewable energy systems for off grid rural electrification of remote area. Renewable Energy, 35, 1342-1349.

[24] Mendes, G., Ioakimidis, C., \& Ferrao, P. (2011) On the planning and analysis of Integrated Community Energy Systems: A review and survey of available tools. Renewable and Sustainable Energy Reviews, 15, 4836- 4854.

[25] Gupta, A., Saini, R. P., \& Sharma, M. P. (2011) Modelling of hybrid energy system Part I: Problem formulation and model Development. Renewable Energy, 36, 459-465.

[26] Ranaboldo, M., Domenech, B., Vilar, D., Ferrer-Martí, L., García-Villoria, A., \& Pastor, R. (2014) Renewable energy projects to electrify rural communities in Cape Verde. Applied Energy, 118, 280-291. 
[27] Silver Edward, A. (2004) An overview of heuristic solution methods. The Journal of the Operational Research Society, 55, 936-956.

[28] Mitra, I. (2008) Optimum utilization of renewable energy for electrification of small islands in developing countries. Ph.D. dissertation in Renewable Energies and Energy Efficiency, 11th ed. Vol. 11, J.Schmid, Ed. Univ. Kassel, 7-213.

[29] Ranaboldo, M., Ferrer-Martí, L., García-Villoria A., \& Pastor, R. (2013) Heuristic indicators for the design of community off-grid electrification systems based on multiple renewable energies. Energy, 50, 501-12.

[30] Sridhar, V., Park, J. S., \& Gavish, B. (2000) LP-based heuristic algorithms for interconnecting token rings via source routing bridges. Journal of Heuristics. 6, 149-166.

[31] Fischetti, M., Polo, C., \& Scantamburlo, M. (2004) A Local Branching Heuristic for Mixed-Integer Programs with 2-Level Variables, with an Application to a Telecommunication Network Design Problem. Networks, 44, 61-72.

[32] Avella, P., Villacci, D., \& Sforza, A. (2005) A Steiner arborescence model for the feeder reconfiguration in electric distribution networks. European Journal of Operational Research, 164, 505-509.

[33] Gueymard, C.A., \& Wilcox, S. M (2011) Assessment of spatial and temporal variability in the US solar resource from radiometric measurements and predictions from models using ground-based or satellite data. Solar Energy, 85, 1068-1084.

[34] Dillenberger, Ch., Escudero, L. F., Wollensak, A., \& Zhang, W. (1994) On Practical Resource Allocation for Production Planning and Scheduling with Period Overlapping Setups. European Journal of Operational Research, 75, 275-286.

[35] Sniedovich, M., \& Voß, S. (2006) The corridor method: a dynamic programming inspired metaheuristic. Control and Cybernetics, 35, 551-578.

[36 Maniezzo, V., \& Voß, S. (2009) Matheuristics: Hybridizing Metaheuristics and Mathematical Programming. Annals of Information Systems, 11.

[37] Caserta, M., Voß, S., \& Sniedovich, M. (2011) Applying the corridor method to a blocks relocation problem. OR Spectrum, 33, 915-929.

[38] Caserta, M., \& Voß, S. (2009) Corridor selection and fine tuning for the corridor method. Lecture Notes on Computer Science 5851, 163-175.

[39] Mortensen, N., \& Peterson, E. (1998) Influence of topographical input data on the accuracy of wind flow modeling in complex terrain. European Wind Energy Conference \& Exhibition, Dublin (Ireland). 